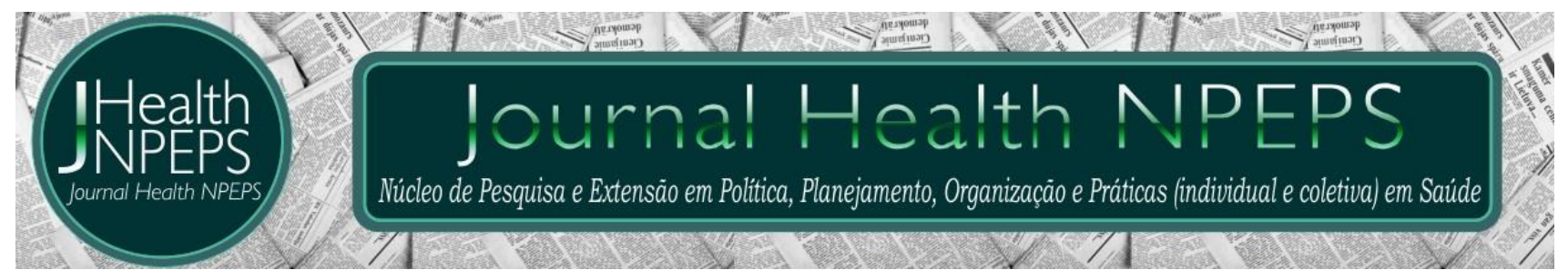

http://dx.doi.org/10.30681/252610104802

ARTIGO ORIGINAL

\title{
Programa psicoterapêutico de prevenção do suicídio em adolescentes: estudo de Delphi
}

Psychotherapeutic program to prevent suicide in adolescents: a Delphi study

\section{Programa psicoterapéutico para prevenir el suicidio en adolescentes: un estudio Delphi}

\author{
Rosa Maria Simões ${ }^{1}$, José Carlos Santos ${ }^{2}$, Maria Júlia Martinho
}

\begin{abstract}
RESUMO
Objetivo: sistematizar o conteúdo de um programa de intervenção psicoterapêutica de prevenção de suicídio em adolescentes com comportamento suicidário. Método: estudo de Delphi, com recurso a grupo de peritos, para validar o conteúdo do programa de intervenção psicoterapêutica proposto. Foram incluídos como peritos, enfermeiros especialistas em enfermagem de saúde mental e psiquiátrica, com título atribuído pela ordem dos enfermeiros e com experiência profissional, de pelo menos dois anos, com adolescentes com comportamento suicidário. 0 instrumento de recolha de dados, sob a forma de questionário, foi divulgado pela ordem dos enfermeiros. Aos peritos foi pedido que assinalassem a sua concordância com o conteúdo do programa através de uma escala tipo likert, em duas rondas. Resultados: o programa final, validado pelos peritos, tem a duração de 10 a 15 sessões, ajustadas consoantes à avaliação clínica e do contexto. A família participa na primeira e sétima sessão. A última sessão será de follow-up e a avaliação do programa é realizada no início, final e na sessão de followup. Conclusão: o programa de intervenção psicoterapêutica de prevenção de suicídio em adolescentes com comportamento suicidário obteve concordância dos peritos em todas as sessões, procedimentos e aspectos gerais.

Descritores: Adolescente; Suicídio; Intervenção na Crise; Prevenção Secundária.
\end{abstract}

\section{ABSTRACT}

Objective: to systematize the content of a psychotherapeutic intervention program to prevent suicide in adolescents with suicidal behavior. Method: Delphi study, using a group of experts, to validate the content of the proposed psychotherapeutic intervention program. Nurses specialized in mental health and psychiatric nursing, with

\footnotetext{
${ }^{1}$ Enfermeira. Mestre e. Doutoranda em Ciências de Enfermagem. Instituto de Ciências Biomédicas de Abel Salazar Universidade do Porto. Porto, Portugal. E-mail: rosasimoes18@gmail.com ORCID ID: https://orcid.org/0000-00026861-4928 Autor para correspondência - Endereço: Praceta Prof. Mota Pinto, 3000-075 Coimbra, Portugal.

${ }^{2}$ Enfermeiro. Doutor em Saúde Mental e Psiquiatria. Professor Coordenador na Escola Superior de Enfermagem de Coimbra. Coimbra, Portugal. E-mail: jcsantos@esenfc.pt ORCID ID: https://orcid.org/0000-0003-1574-972X

${ }^{3}$ Enfermeira. Doutora em Ciências de Enfermagem. Professor Adjunto Escola Superior de Enfermagem do Porto. Porto, Portugal. E-mail: julia@esenf.pt ORCID ID http://orcid.org/0000-0002-5233-8324
}

Este artigo está licenciado sob forma de uma licença Creative Commons Atribuição 4.0 Internacional, que permite uso irrestrito, distribuição e reprodução em qualquer meio, desde que a publicação original seja corretamente citada 
a title attributed by the order of nurses and professional experience of at least two years, with adolescents with suicidal behavior were included as experts. The data collection instrument, in the form of a questionnaire, was released by the nurses' order. The experts were asked to indicate their agreement with the content of the program using a likert scale, in two rounds. Results: the final program, validated by the experts, lasts 10 to 15 sessions, adjusted according to clinical and contextual assessment. The family participates in the first and seventh session. The last session will be a follow-up and the program evaluation is carried out at the beginning, at the end and in the follow-up session. Conclusion: the suicide prevention psychotherapeutic intervention program for adolescents with suicidal behavior obtained agreement from the experts in all sessions, procedures and general aspects.

Descriptors: Adolescent; Suicide; Crisis Intervention; Secondary Prevention.

\section{RESUMEN}

Objetivo: sistematizar el contenido de un programa de intervención psicoterapéutica para prevenir el suicidio en adolescentes con conducta suicida. Método: estudio Delphi, con un grupo de expertos, para validar el contenido del programa de intervención psicoterapéutico propuesto. Se incluyeron como expertos enfermeros especializados en salud mental y enfermería psiquiátrica, con título atribuido por orden de enfermeros y experiencia profesional de al menos dos años, con adolescentes con conducta suicida. El instrumento de recolección de datos, en forma de cuestionario, fue liberado por orden de las enfermeras. Se pidió a los expertos que indicaran su acuerdo con el contenido del programa mediante una escala Likert, en dos rondas. Resultados: el programa final, validado por los expertos, tiene una duración de 10 a 15 sesiones, ajustadas según valoración clínica y contextual. La familia participa en la primera y séptima sesión. La última sesión será de seguimiento y la evaluación del programa se realiza al inicio, final y en la sesión de seguimiento. Conclusión: el programa de intervención psicoterapéutica de prevención del suicidio para adolescentes con conducta suicida obtuvo el acuerdo de los expertos en todas las sesiones, procedimientos y aspectos generales.

Descriptores: Adolescente; Suicidio; Intervención en la Crisis; Prevención Secundaria.

\section{INTRODUÇÃO}

O suicídio é a segunda causa de morte nos adolescentes dos 15 aos 19 anos de idade sendo responsável por $85 \%$ das mortes nesta faixa etária em todo o mundo, com evidências relativas a um aumento do número de $\operatorname{casos}^{1}$. Como agravante, é um fenômeno caraterizado por uma natureza repetitiva que o torna num dos maiores preditores de comportamentos futuros e de eventual morte por suicídio ${ }^{2}$. 0 risco de repetição é acrescido nos três a seis meses seguintes ao comportamento e nos nove anos após uma tentativa de suicídio cerca de 3 a 12\% dos indivíduos terão morrido por suicídio ${ }^{2-4}$.

Cientes de toda a complexidade inerente ao fenômeno em análise, antes da concessão do programa psicoterapêutico de prevenção do suicídio em adolescentes com comportamentos suicidários foi 
desenvolvida uma revisão integrativa da literatura que teve a finalidade de identificar a evidência científica sobre as intervenções psicoterapêuticas dirigidas a adolescentes com comportamentos suicidários e conhecer a eficácia dessas intervenções ao nível da ideação suicida, depressão e repetição do comportamento suicidário ${ }^{5}$.

Esta revisão integrativa da literatura foi realizada com pesquisa em bases de dados bibliográficas online, com os descritores de pesquisa selecionados, no período de publicação de janeiro de 2007 a dezembro de 2016, em inglês, com referências disponíveis e artigos em texto integral. Foram incluídos 10 estudos com adolescentes com comportamento suicidário, alvo de intervenção psicoterapêutica. A operacionalização dos programas que obtiveram resultados positivos remete para 0 uso de intervenções psicoterapêuticas majoritariamente do tipo cognitivo comportamental, com psicoeducação e treino de habilidades individuais e familiares, intervenção comunitária, terapia dialético comportamental e terapia da mentalização, em sessões individuais e unifamiliares. A duração dos programas é variável assim como o número de sessões, com periodicidade de 12 semanas a 12 meses, de 8 a 42 sessões, predominantemente realizadas em 60 minutos. A inclusão da família aconteceu em todos os programas, mas de forma heterogênea, com participação exclusiva numa sessão do programa, atribuição de terapeutas exclusivos para familiares, participação nas visitas domiciliárias, sessão multifamiliar ou terapia familiar. Quanto ao follow-up verificou-se em três dos seis programas, sendo também heterogêneo em termos de metodologia, assim como a avaliação dos programas ${ }^{5}$. Perante estes resultados, mais se evidenciou a complexidade e necessidade de desenvolvimento deste programa de intervenção psicoterapêutica.

No entanto, a prevenção dos comportamentos suicidários deve atender às opiniões, necessidades e expectativas dos adolescentes para daí serem emanadas orientações sistematizadas de intervenção mais congruentes $^{6}$. Para tal, procedeu-se à realização de estudo exploratório descritivo de natureza qualitativa, com 33 adolescentes com comportamento suicidário e internamento em serviço de pedopsiquiatria. Este estudo buscou conhecer a opinião dos adolescentes relativamente ao internamento e conhecer as expectativas de 
envolvimento da família e de continuidade de cuidados ${ }^{7}$.

Constataram que relativamente aos aspectos mais significativos do internamento, os adolescentes destacam a importância do acompanhamento psicológico, dos profissionais e das atividades ocupacionais ${ }^{7}$. Ainda assim, os conselhos para melhorar o atendimento são essencialmente dirigidos à gestão do ambiente e às intervenções que consideram em número insuficiente $\mathrm{e}$ pouco dirigidas à aquisição de novas competências para prevenção de novo comportamento suicidário7. A família é descrita como maioritariamente presente e como expectativa revelam que gostariam que mantivesse o apoio atual e que, se possível, recebessem, também eles, apoio especializado. Para a preparação para a alta destacam a necessidade de manter o contato após a alta com os enfermeiros ${ }^{7}$.

Encontrou-se justificativa para a realização do presente estudo no fato de não existir em Portugal qualquer intervenção de enfermagem, estruturada, sistematizada e congruente com os problemas e expectativas dos adolescentes com comportamento suicidário. Desta forma, e seguindo as premissas anteriormente apresentadas, o presente estudo teve como objetivo sistematizar o conteúdo de um programa de intervenção psicoterapêutica de prevenção de suicídio em adolescentes com comportamento suicidário.

\section{MÉTODO}

0 método selecionado para realizar este estudo foi a técnica de Delphi, que seguiu as quatro etapas metodológicas: revisão da literatura, fase pré-Delphi, fase de preparação e fase Delphi ${ }^{8}$. Todas estas etapas foram realizadas na zona centro de Portugal, à exceção da fase Delphi que incluiu todos os enfermeiros especialistas em enfermagem de saúde mental e psiquiátrica de Portugal. A realização total do estudo decorreu de Maio de 2019 a Junho de 2020, num total de 13 meses.

A revisão da literatura e construção da proposta do programa psicoterapêutico se concretizou com base na revisão integrativa da literatura e com base na investigação realizada com adolescentes com comportamento suicidário e internamento em unidade de pedopsiquiatria, em hospital pediátrico da região centro de Portugal ${ }^{5,7}$. Daqui decorreram a seleção das estratégias que constam em, pelo menos $50 \%$ dos estudos analisados e a definição dos 
objetivos, dos procedimentos, das atividades a desenvolver, assim como das questões relativas à população-alvo, duração do programa e sua avaliação.

O programa proposto tem como objetivo prevenir a repetição de comportamentos suicidários, diminuir 0 risco de suicídio, melhorar as competências de resolução de problemas e promover a esperança e razões para viver. 0 envolvimento da família é concretizado na primeira e sétima sessão e a avaliação do programa será realizada com recurso a um questionário aplicado no início, no final e na sessão de followup. Este questionário inclui: índice NGASR (The Nurses Global Assessment of Suicide Risk) para avaliação do risco de suicídio"; inventário de razões para viver $^{10}$; escala de esperança ${ }^{11}$; e escala toulosiana de coping ${ }^{12}$.

$\mathrm{Na}$ fase pré-Delphi foi realizado o pré-teste, com aplicação do questionário a um grupo de quatro enfermeiros, especialistas em enfermagem de saúde mental e psiquiátrica com experiência em prevenção do suicídio em adolescentes.

$\mathrm{Na}$ fase de preparação selecionou os peritos, com base nos seguintes critérios: ser enfermeiro especialista em enfermagem de saúde mental e psiquiátrica, com título atribuído pela ordem dos enfermeiros e ter experiência profissional, de pelo menos dois anos, com adolescentes com comportamento suicidário. Foi ainda concluída a proposta de programa psicoterapêutico com os contributos do pré-teste e obtenção de parecer favorável de comissão de ética (Parecer $\mathrm{n}^{\circ} \quad$ P601/06-2019). Todos os procedimentos seguiram os padrões éticos exigidos.

Já na fase Delphi, a operacionalização do método Delphi se concretiza através de rondas de questionários aplicados aos peritos, até obtenção de consenso. Assim, o questionário foi constituído por questões relativas à caraterização sociodemográfica e profissional dos peritos e por todos os procedimentos de todas as sessões que compõem a proposta de programa psicoterapêutico. No final do questionário, foi permitido aos peritos expressar a sua opinião sobre o programa e acrescentar sugestões.

Este questionário foi enviado por meio de newsletter, pela Ordem dos Enfermeiros a todos os enfermeiros especialistas em enfermagem de saúde mental e psiquiátrica, portugueses. Os critérios estabelecidos para os participantes no grupo de peritos foram: ser enfermeiro especialista em 
enfermagem de saúde mental e psiquiátrica com título atribuído pela Ordem dos Enfermeiros e ter experiência profissional, de pelo menos dois anos, com adolescentes com comportamento suicidário. A estes foi solicitado que assinalassem a sua concordância, com o conteúdo do programa, através de uma escala tipo likert, composta por cinco níveis, desde o discordo totalmente até ao concordo totalmente.

A recolha de dados na primeira ronda ocorreu em Janeiro de 2020 e a segunda ronda entre Maio e Junho de 2020.

O tratamento de dados foi realizado através dos operativos Google Docs e Statistical Package for the Social Sciences (SPSS) - versão 25, com cálculo da distribuição de frequências e das medidas de tendência central. No entanto, antes da análise dos dados obtidos foi definido o nível de consenso ${ }^{13}$.

Para concordância positiva as respostas tiveram que se situar nos níveis concordo e concordo totalmente, obtendo-se consenso perfeito para $100 \%$ de respostas nestes níveis; consenso forte - respostas com nível de consenso superior ou igual a $90 \%$ e inferior a $100 \%$; consenso moderado - respostas com nível de consenso superior ou igual a $80 \%$ e inferior a 90\%; consenso fraco as respostas teriam que se situar com nível de consenso inferior a $80 \%$ e significariam não concordância, levando a nova ronda.

\section{RESULTADOS}

A amostra de peritos selecionada foi constituída por 27 elementos que cumpriram os critérios de seleção. Quanto à sua caraterização segundo as variáveis sóciodemográficas selecionadas, é uma amostra maioritariamente constituída por elementos do gênero feminino $(66,70 \%)$, com média de idade de 44,15 anos, prevalecendo casados e/ou em união de fato $(66,7 \%)$. Apresentam média de 22,26 anos de serviço e em sua maioria exercem funções em cuidados de saúde diferenciados $(63,00 \%)$.

0 grupo de peritos considera muito importante $(44,40 \%)$ ou extremamente importante $(55,60 \%)$ a sistematização deste programa de intervenção psicoterapêutica, e a quase totalidade $(96,30 \%)$ considera que a prevenção do suicídio em adolescentes implica uma relação terapêutica entre o enfermeiro e o cliente e um setting próprio $(88,90 \%)$. 
Os peritos atribuíram consenso perfeito e consenso elevado aos objetivos definidos, e a todos os dados obrigatórios para a prescrição da intervenção psicoterapêutica, com exceção do dado obrigatório proposto "impulsividade" que obteve consenso moderado $(81,40 \%)$.

A primeira ronda decorreu de 3 a 20 de Janeiro de 2020. Obtiveram-se 106 respostas e pela aplicação dos critérios de seleção foi identificado um grupo de 27 peritos. Os níveis de consenso obtidos relativamente aos aspectos gerais do programa constam na Tabela 1 , e se referem ao somatório dos níveis "concordo" com "concordo totalmente".

Em relação aos procedimentos das sessões, os níveis de consenso obtidos estão patentes na Tabela 2, que pela necessidade de sintetização se referem apenas aos procedimentos principais. Nesta Tabela, pode verificar que o procedimento "efetuar separação física" obteve o nível de consenso mais baixo com $85,20 \%$ e os itens "negociar os objetivos do programa”, “explorar motivações para o suicídio", "negociar contrato não suicídio" e "promover reflexão acerca do impacto do comportamento suicidário, em si e nos outros" obtiveram nível de consenso de $88,90 \%$. Todos os restantes apresentam nível de consenso superior a 90\% (Tabela 2).

Considerando o nível de consenso obtido para as questões relativas aos aspectos gerais do programa $(70,30 \%)$ foi realizada segunda ronda específica para estas questões. Após apresentar aos peritos os resultados obtidos na primeira ronda, foram novamente colocadas questões referentes aos aspectos gerais do programa. A segunda ronda decorreu de 28 de Maio a 7 de Junho de 2020 e o grupo de peritos foi constituído por 22 enfermeiros que preenchiam os critérios de seleção e que terão respondido à primeira ronda.

Tabela 1 - Níveis de consenso obtidos nos aspectos gerais do programa, na primeira ronda.

\begin{tabular}{lc}
\hline Aspectos gerais do programa & Nível consenso \\
\hline O programa proposto tem a duração de quatro a cinco semanas (duas a três sessões & $70,30 \%$ \\
por semana, com duração de 60 minutos cada) & $70,30 \%$ \\
O programa proposto tem no mínimo nove sessões & $70,30 \%$ \\
O programa proposto tem no máximo doze sessões & \\
\hline
\end{tabular}


Tabela 2 - Níveis de consenso obtidos nos procedimentos das sessões do programa.

\begin{tabular}{|c|c|c|}
\hline Sessões & Procedimentos & Nível consenso \\
\hline \multirow[t]{4}{*}{ Sessão 1} & Avaliar condições de segurança para a pessoa e terceiros & $96,30 \%$ \\
\hline & Promover envolvimento familiar & $96,30 \%$ \\
\hline & Negociar objetivos do programa & $88,90 \%$ \\
\hline & Obter consentimento informado e negociar contrato terapêutico & $96,30 \%$ \\
\hline \multirow[t]{4}{*}{ Sessão 2} & Explorar motivações para o suicídio & $88,90 \%$ \\
\hline & $\begin{array}{l}\text { Identificar, por escrito, estímulos que aumentam o comportamento } \\
\text { suicidário }\end{array}$ & $96,30 \%$ \\
\hline & Discutir objetivos do comportamento, sem julgamento & $92,60 \%$ \\
\hline & Negociar contrato não suicídio & $88,90 \%$ \\
\hline \multirow[t]{3}{*}{ Sessão 3} & $\begin{array}{l}\text { Promover reflexão acerca do impacto do comportamento suicidário, } \\
\text { em si e nos outros }\end{array}$ & $88,90 \%$ \\
\hline & Treinar estratégias alternativas de resolução de problemas & $96,30 \%$ \\
\hline & Elaborar Plano de Segurança & $96,30 \%$ \\
\hline \multirow[t]{3}{*}{ Sessão 4} & Explorar fatores protetores e reforçá-los & $92,60 \%$ \\
\hline & Identificar os fatores de risco & $92,60 \%$ \\
\hline & Reforcar estratégias de resolucão de problemas & $96,30 \%$ \\
\hline \multirow[t]{3}{*}{ Sessão 5} & Rever plano segurança & $96,30 \%$ \\
\hline & Motivar a pessoa a definir um projeto de vida & $96,30 \%$ \\
\hline & $\begin{array}{l}\text { Ajudar a examinar recursos disponíveis (ex.: apoio familiar, } \\
\text { comunitário,...) }\end{array}$ & $96,30 \%$ \\
\hline \multirow[t]{4}{*}{ Sessão 6} & Elaborar Kit de Esperança & $92,60 \%$ \\
\hline & Enunciar razões para viver & $96,30 \%$ \\
\hline & Efetuar reforços positivos & $96,30 \%$ \\
\hline & Manter restrição de acesso a meios letais & $92,60 \%$ \\
\hline \multirow[t]{3}{*}{ Sessão 7} & $\begin{array}{l}\text { Ajudar a examinar recursos disponíveis (apoio familiar, apoio de } \\
\text { amigos, apoio escolar...) }\end{array}$ & $92,60 \%$ \\
\hline & Promover envolvimento familiar & $96,30 \%$ \\
\hline & Executar psicoeducacão familiar & $96,30 \%$ \\
\hline \multirow[t]{3}{*}{ Sessão 8} & Discutir plano de segurança & $96,30 \%$ \\
\hline & $\begin{array}{l}\text { Promover continuidade de cuidados ao nível dos cuidados de saúde } \\
\text { primários e ao nível especializado }\end{array}$ & $96,30 \%$ \\
\hline & $\begin{array}{l}\text { Clarificar formas de contato futuro (sms, telefonema, e-mail, } \\
\text { carta,...) }\end{array}$ & $96,30 \%$ \\
\hline \multirow[t]{3}{*}{ Sessão 9} & Avaliar o trabalho e fazer síntese dos resultados & $96,30 \%$ \\
\hline & Esclarecer dúvidas & $96,30 \%$ \\
\hline & Efetuar separação física & $85,20 \%$ \\
\hline & Rever Plano de Segurança e Kit de Esperança & $92,60 \%$ \\
\hline \multirow{3}{*}{ (Follow-up) } & Avaliar impacto da mobilização familiar & $92,60 \%$ \\
\hline & Avaliar apoio dos recursos comunitários & $92,60 \%$ \\
\hline & Resumo e avaliacão da recuperacão & $92,60 \%$ \\
\hline
\end{tabular}

$\mathrm{Na}$ Tabela 3 constam os somatório dos níveis concordo e resultados obtidos e que se referem ao concordo totalmente.

Tabela 3 - Níveis de consenso obtidos nos aspectos gerais do programa, na segunda ronda.

\begin{tabular}{ll}
\hline Aspectos gerais do programa & Nível consenso \\
\hline O programa proposto tem a duração de sete a oito semanas (duas a três sessões por & $86,36 \%$ \\
semana, com duração de 60 minutos cada) & $86,36 \%$ \\
O programa proposto tem no mínimo 10 sessões & $81,81 \%$ \\
O programa proposto tem no máximo 15 sessões & \\
\hline
\end{tabular}




\section{DISCUSSÃO}

Todas as sessões e procedimentos do programa proposto reuniram concordância positiva por parte do grupo de peritos logo na primeira volta da técnica de Delphi, assim todos os procedimentos que o constituem foram aprovados com consenso forte. As questões que obtiveram consenso fraco e consequentemente não concordância pelo grupo de peritos, foram as relativas aos aspectos gerais do programa, nomeadamente no que se refere à duração e número de sessões, levando a uma segunda ronda dirigida a estas questões.

Pelos resultados obtidos na segunda ronda optou por definir um programa com duração de sete a oito semanas, para um número mínimo de 10 sessões e um número máximo de 15 sessões com consenso moderado. Foi salvaguardado e reforçado pelos peritos que mediante avaliação clínica, este número de sessões deverá ser ajustado ao indivíduo, às suas necessidades e ao contexto de implementação.

0 nível de consenso moderado obtido ajusta às evidências existentes e relativas a heterogeneidade das intervenções psicoterapêuticas em termos de intensidade e de duração dos programas, podendo variar de duração entre uma e duas semanas até um período de 36 meses, sendo a maioria desenvolvidas no período de dois a quatro meses ${ }^{4,5,13,14}$. Estas variações são justificadas de acordo com a tipologia, procedimentos utilizados e os objetivos estabelecidos para os programas.

Quanto ao número de sessões por semana, a maioria dos estudos analisados aponta para uma ou duas sessões por semana ${ }^{5,14}$. Justifica-se a opção por propor a realização de duas a três sessões por semana com base na perspetiva da prescrição do programa psicoterapêutico de prevenção do suicídio o mais precoce e de forma intensa possível, em contexto de internamento. Esta proposta, que obteve consenso moderado por parte dos peritos, apoia-se na evidência que as intervenções psicoterapêuticas de transição, como as passíveis de desenvolvimento no internamento psiquiátrico, parecem ser as mais promissoras para reduzir os resultados relacionados com o suicídio e melhorar a adesão ao tratamento ${ }^{7,15}$. A implementação de estratégias de prevenção de comportamento suicidário precocemente irá garantir acompanhamento psicoterapêutico imediatamente após o início do 
internamento, mantendo-se este acompanhamento após a alta6,16,17.

As sessões e procedimentos no âmbito do programa psicoterapêutico de prevenção do suicídio em adolescentes com comportamentos suicidários serão realizadas individualmente, com 0 envolvimento da família na primeira sessão e com sessão psicoeducativa familiar na sétima sessão. Esta proposta se fundamenta nas evidências científicas que apontam para uma maior efetividade das intervenções individuais e sistémicas em detrimento das intervenções em grupo 5,18,19,20,21. A proposta apresentada relativamente ao envolvimento da família obteve consenso elevado por parte dos peritos, o que corrobora as evidências disponíveis, em que o envolvimento da família é consensual ${ }^{5,22,23}$. Também os adolescentes com comportamento suicidário elegeram a família, amigos e pessoas de confiança como principal fator protetor de novo comportamento, e manifestaram ainda a necessidade de ver os pais apoiados do ponto de vista psicoterapêutico ${ }^{7}$.

A sessão de follow-up também obteve consenso elevado por parte dos peritos e foi também delineada com base nos resultados do estudo qualitativo realizado com adolescentes e na revisão integrativa. Para os adolescentes é crucial manter contato com os profissionais do serviço de pedopsiquiatria e na revisão verificou-se sessão de follow-up em $50 \%$ dos estudos com resultados positivos $5,7,15$.

Relativamente aos momentos de avaliação propostos (antes, final e follow-up), o grupo de peritos demonstra concordância positiva e nas sugestões três peritos reforçam positivamente 0 timing do follow-up (cerca de um mês), por entenderem que uma avaliação mais estreita no tempo pode ser mais vantajosa.

O presente estudo apresenta algumas limitações como a homogeneidade no grupo de peritos selecionados, justificada pela complexidade do tema e pela motivação para que seja um programa implementado por enfermeiros. A ambiguidade dos autores na definição da variação dos níveis de consenso também se configurou como uma limitação.

Quanto à relevância para a prática clínica e pelos resultados obtidos, entende-se que o estudo realizado apresenta grande relevância já que fornece um instrumento construído com base em evidências científicas retiradas de várias fontes, sistematizado e validado por peritos. 
Como recomendações priorizou a validação clínica deste programa e o desenvolvimento de uma intervenção psicoterapêutica dirigida à família, assim como a elaboração de protocolos de cooperação e colaboração estruturada entre cuidados de saúde especializados e cuidados de saúde primários.

\section{CONCLUSÃO}

Todas as sessões, procedimentos e questões relativas aos aspectos gerais do programa proposto reuniram concordância positiva no grupo de peritos. Desta forma, o programa psicoterapêutico de prevenção do suicídio em adolescentes com comportamentos suicidário proposto terá a duração de sete a oito semanas, mínimo 10 sessões e máximo 15 sessões, com duas a três sessões por semana e duração de 60 minutos cada. As sessões são individuais, com o envolvimento da família na primeira e sétima sessão, sendo a última sessão de follow-up. A avaliação do programa será realizada com recurso a questionário composto por instrumentos de medida validados para a população portuguesa e aplicados antes, no final e no follow-up da intervenção. Definido que deve ser implementado imediatamente após a avaliação do adolescente com comportamento suicidário e preferencialmente em contexto de internamento.

Ainda assim, mediante avaliação clínica, o programa é flexível e passível de ser aplicado e adaptado ao contexto e participantes. Para a sua implementação e pela necessidade de competências específicas em enfermagem de saúde mental e psiquiátrica e em suicidologia, entendese que deve ser preferencialmente implementado por enfermeiros especialistas nesta área, com formação e treinamento em suicidologia.

\section{REFERÊNCIAS}

1. World Health Organization. Suicide in the world: global health estimates [Internet]. Geneva: WHO; 2019 [cited 2020 Abr 22]; 32 p. Available from: https://apps.who.int/iris/bitstream/h andle/10665/326948/WHO-MSD-MER-

\section{3-eng.pdf}

2. Wright-Hughes A, Graham E, Farrin A, Collinson $M$, Boston $P$, Eisler $I$, et al. Self-harm intervention: family therapy (SHIFT), a study protocol for a randomised controlled trial of family therapy versus treatment as usual for young people seen after a second or subsequent episode of self-harm. 
Trials. 2015; 16(501).

3. McCauley E, Berk MS, Asarnow JR, Adrian M, Cohen J, Korslund k, et al. Efficacy of Dialectical Behavior Therapy for Adolescents at High Risk for Suicide: A Randomized Clinical Trial. JAMA Psychiatry. 2018; 75(8):777-785.

4. Ougrin D, Tranah T, Stahl D, Moran P, Asarnow JR. Therapeutic interventions for suicide attempts and self-harm in adolescents: systematic review and meta-analysis. J Am Acad Child Adolesc Psychiatry. 2015; 54(2):97107.

5. Simões R, Santos JC, Martinho MJ. Eficácia das intervenções psicoterapêuticas dirigidas a adolescentes com comportamento suicidário: revisão integrativa da literatura. Rev Enferm Ref. 2019; serlV:139-48.

6. Centre of Research Excellence in Suicide Prevention (NHMRC). Care after a suicide attempt. Sydney: National Mental Health Commission; 2015.

7. Simoes R, Santos JC, Martinho MJ. Adolescents with suicidal behaviours: qualitative study about assessment of Inpatient Service and Transition to Community. J Psychiatr Ment Health Nurs. No Prelo; 2020.
8. López-Gómez E. O método delphi na atual investigação em educação: uma revisão teórica e metodológica. Educação XX1. 2018; 21(1):17-40.

9. Façanha J, Santos JC, Cutcliffe J. Assessment of suicide risk: validation of the nurses' global assessment of suicide risk index for the portuguese population. Arch Psychiatr Nurs. 2016; 30(4):470-475.

10. Matias J, Santos JC. Inventário de razões para viver: contributos para a validação para a população não clínica portuguesa. Rev Port Enferm Saúde Ment. 2014; (11):9-14.

11. Barros-Oliveira JH. Felicidade, optimismo, esperança e perdão em jovens, adultos e idosos. Psychologica. 2010; 52(1):123-44.

12. Tap P, Costa ES, Alves MN. Escala toulousiana de coping (etc): estudo de adaptação à população portuguesa. Psic saúde doenças. 2005; 6(1):47-56.

13. Scarparo AF, Laus AM, Azevedo ALCS, Freitas MRI, Gabriel CS, Chaves LDP. Reflexões sobre a técnica delphi em pesquisa na enfermagem. Rev Rene. 2012; 13(1):242-51.

14. Shand F, Vogl L, Robinson J. Improving patient care after a suicide attempt. Australas 
Psychiatry. 2018; 26(2):145-148.

15. Méndez-Bustos $P$, Calati R, RubioRamírez F, Olié $\mathrm{E}$, Courtet $\mathrm{P}$, LopezCastroman J. Effectiveness of psychotherapy on suicidal risk: a systematic review of observational studies. Front Psychol. 2019; 10:277.

16. Newton AS, Hartling L, Soleimani A, Kirkland S, Dyson MP, Cappelli M. A systematic review of management strategies for children's mental health care in the emergency department: update on evidence and recommendations for clinical practice and research. Emerg Med J. 2017; 34:376-384.

17. Mehlum L, Ramberg M, Tørmoen AJ, Haga E, Diep LM, Stanley BH, et al. Dialectical Behavior Therapy Compared With Enhanced Usual Care for Adolescents With Repeated Suicidal and Self-Harming Behavior: Outcomes Over a One-Year FollowUp. J Am Acad Child Adolesc Psychiatry. 2016; 55(4):295-300.

18. Berk MS, Starace NK, Black VP, Avina C. Implementation of Dialectical Behavior Therapy with Suicidal and Self-Harming Adolescents in a Community Clinic. Arch Suicide Res. 2020; 24(1):64-81.

19. Hazell P, Martin G, Mcgill K, Kay T, Wood A, Trainor G, et al. Group therapy for repeated deliberate selfharm in adolescents: failure of replication of a randomized trial [Internet]. J Am Acad Child Adolesc Psychiatry. 2009; 48(6):662-670.

20. Glenn CR, Esposito EC, Porter AC, Robinson DJ. Evidence Base Update of Psychosocial Treatments for SelfInjurious Thoughts and Behaviors in Youth. J Clin Child Adolesc Psychology. 2019; 48:(3):357-392.

21. Robinson J, Bailey E, Witt K, Stefanac N, Milner A, Currier D, Pirkis $\mathrm{J}$, et al. What Works in Youth Suicide Prevention? A Systematic Review and Meta-Analysis. EClinicalMedicine. 2018; 28(4-5):5291.

22. Hawton K, Witt KG, Salisbury TL, Arensman E, Gunnell D, Hazell P, et al. Pharmacological interventions for self-harm in adults. Cochrane Database Syst Rev. 2015; (7):CD011777.

23. Liba YHAO, Lemes AG, Oliveira PR, Nascimento VF, Fonseca PIMN, Volpato RJ, et al. Percepções dos profissionais de enfermagem sobre 0 paciente pós-tentativa de suicídio. J Health NPEPS. 2016; 1(1):109-121. 
Financiamento: Os autores declaram que não houve financiamento.

Conflito de interesses: Os autores declaram não haver conflito de interesses.

\section{Participação dos autores:}

- Concepção: Simões RM, Santos JC, Martinho MJ.

- Desenvolvimento: Simões RM.

- Redação e revisão: Simões RM, Santos JC, Martinho MJ.

Como citar este artigo: Simões RM, Santos JC, Martinho MJ. Programa psicoterapêutico de prevenção do suicídio em adolescentes: estudo de Delphi. J Health NPEPS. 2020; 5(2):75-88.

Submissão: $11 / 08 / 2020$

Aceito: $13 / 11 / 2020$

Publicado: 04/12/2020 